\title{
A Rare Case of Primary Cutaneous Aspergillosis of the Scalp in an Immunocompetent Patient
}

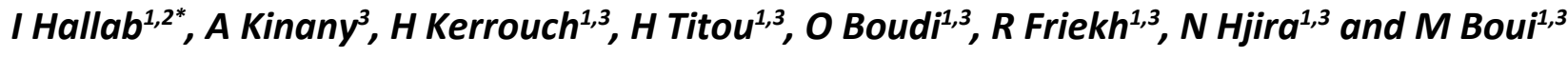 \\ ${ }^{1}$ Dermatology Department, Military Hospital, University Mohammed V, Morocco \\ ${ }^{2}$ Faculty of Medicine and Pharmacy, University Sidi Mohamed Ben Abdellah USMBA, Morocco \\ ${ }^{3}$ Faculty of Medicine and Pharmacy, University Mohammed V, Morocco \\ *Corresponding author: Ihsane Hallab, Dermatology Department, Military Hospital, Mohammed V, 10100, Rabat, \\ Morocco
}

\section{Keywords \\ Aspergillosis, Scalp mycosis, Griseofulvin}

\section{Abreviations \\ HIV: Human Immunodeficiency Virus}

\section{Background}

Aspergillosis is a rare invasive fungal infection caused by numerous species of aspergillus. Few species of Aspergillus are pathogenic in human existence. The most frequent are aspergillus fumigatus and flavus [1]. Cutaneous aspergillosis remain an uncommon form, it may be primary, affecting the site of skin trauma or secondary due to hematogenous spread from a contiguous anatomic location [2]. Here we describe a rare case of primary cutaneous aspergillosis located on the scalp in an immunocompetent host, which continue to be undiagnosed for a long period of time by dermatologists.

\section{Case Report}

A six-year-old boy presented to the dermatology department with a large erythematous lesion on the scalp that has appeared over 1 year period and had gradually increased in size.

There was no history of trauma, invasive procedures, other infections or similar cases in the family. Cutaneous examination of the scalp revealed an erythematous plaque measuring $10 * 10 \mathrm{~cm}$ with significant loss of hair, covered with gray scales (Figure 1, Figure 2 and Figure $3)$.

Routine blood test and viral serologies were normal. Mycological examination and fungal culture demonstrates the presence of 3 agents: Ureaplamsa urelyticum, Mycoplasma hominis and aspergillus. The diagnosis of scalp mycosis due to aspergillosis was given. The patient begun treatment with a course of oral Terbinafine $125 \mathrm{mg}$ per day for six weeks associated with a wet dressing of Ketoconazole ointment. There was significant if improvement in discharge and pruritus and a reduction of the plaque size after 4 weeks of treatment with no recidive.

\section{Discussion}

Scalp mycosis is often due to dermatophytes, mostly Tinea capitis.

However, less frequently, other species may be identified [3]. Aspergillus species are the most ubiquitous fungi, ordinary seen in soil, water, seeds and grains. The respiratory tract is the principal site of entry. Primary cutaneous aspergillosis may existing with violaceous macules, papules, plaques or nodules. The presence of hemorrhagic bullae is possible. Pustular lesions have been rarely reported.

The vast majority of described cases were among patients with hematologic malignancies, organ

Citation: Hallab I, Kinany A, Kerrouch H, Titou H, Boudi O, et al. (2022) A Rare Case of Primary Cutaneous Aspergillosis of the Scalp in an Immunocompetent Patient. Clin Med Img Lib 8:196. doi. org/10.23937/2474-3682/1510196

Accepted: January 24, 2022; Published: January 26, 2022

Copyright: (c) 2022 Hallab I, et al. This is an open-access content distributed under the terms of the Creative Commons Attribution License, which permits unrestricted use, distribution, and reproduction in any medium, provided the original author and source are credited. 


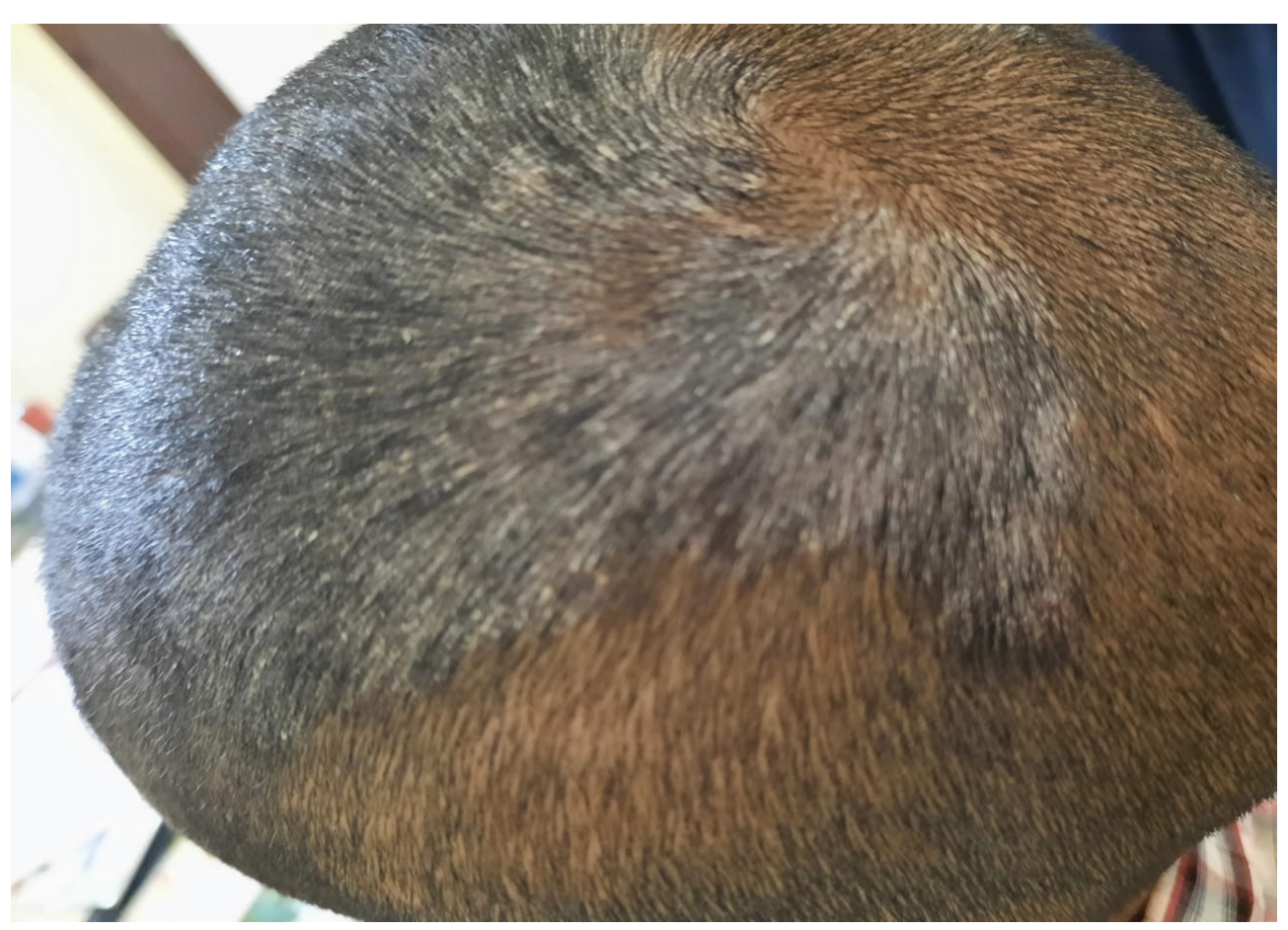

Figure 1: A large plaque covered with gray scales.

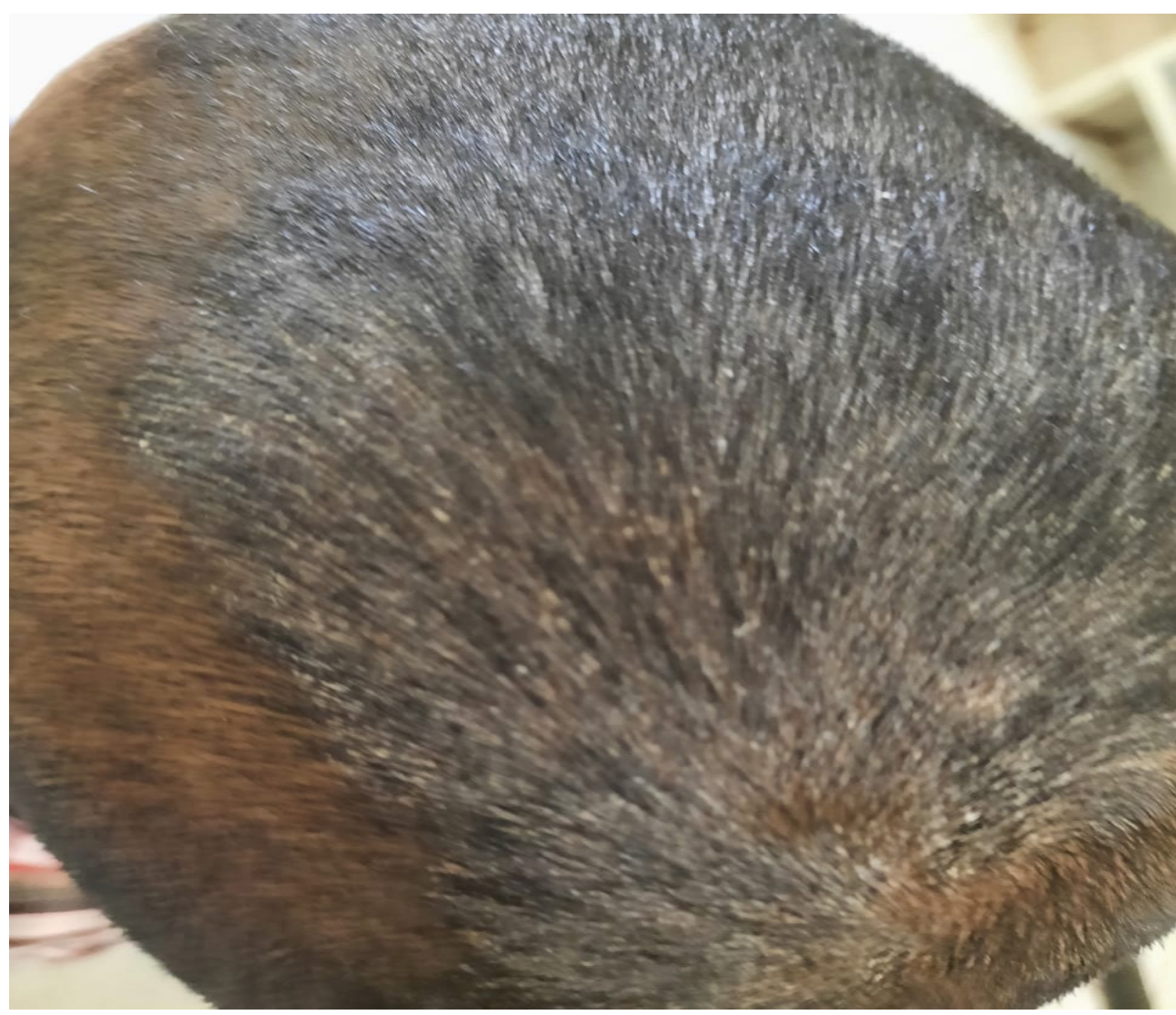

Figure 2: A large plaque covered with gray scales.

transplant, patients under immunosuppressive agent, systemic glucocorticoids or broad-spectrum antibiotics as well as patients with HIV.

Risk factors include trauma to the skin, burns, peripheral and central venous catheters, contaminated materials and inhalation of airborne conidia in construction areas [2].

As for treatment [4], in superficial infection, Terbinafine, Voriconazole, Itraconazole were all effective as well as griseofulvin. Terbinafine has high 


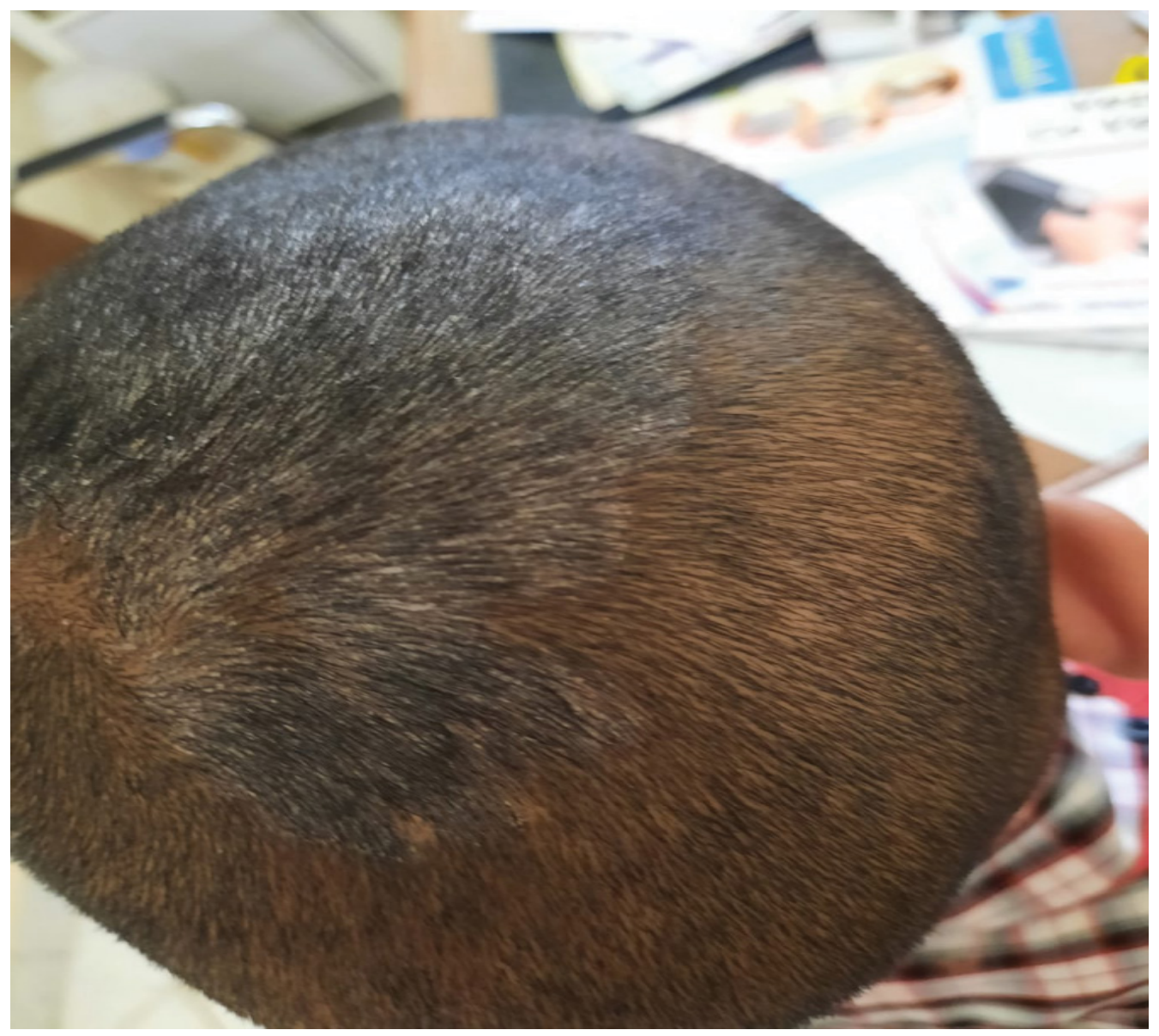

Figure 3: A large plaque covered with gray scales.

concentration in skin, hair, nails and has broad spectrum antifungal activity and remains a good choice. Although there is no gold standard treatment for scalp mycosis of aspergillus in the literature.

\section{Conclusion}

Aspergillus is a chronic colonizer, its manifestations occurs primarily in patients with underlying medical conditions and immunocompromised status. However, to avoid delaying the treatment, diagnosis should be made earlier in front of any resistance.

\section{References}

1. Mohapatra S, Xess I, Swetha JV, Tanveer N, Asati D, et al. (2009) Primary cutaneous aspergillosis due to Aspergillus niger in an immunocompetent patient. Indian $\mathrm{J}$ Med Microbiol 27: 367-370.

2. Ricci RM, Evans JS, Meffert JJ, Kaufman L, Sadkowski LC (1998) Primary cutaneous Aspergillus ustus infection: Second reported case. J Am Acad Dermatol 38: 797-798.

3. Ely JW, Rosenfeld S, Stone MS (2014) Diagnosis and management of tinea infections. Am Fam Physician 90: 702-710.

4. Jia J, Chen M, Mo X, Liu J, Yan F, et al. (2019) The first case report of kerion-type scalp mycosis caused by Aspergillus protuberus. BMC Infect Dis 19: 506. 